\title{
COMPORTAMIENTO DE UN CEMENTO ASFÁLTICO MODIFICADO CON UN DESECHO DE PVC
}

\author{
Fredy Alberto Reyes Lizcano* \\ Catalina Guáqueta Echeona* \\ Laura Melissa Porras Salcedo*** \\ Hugo Alexander Rondón Quintana ${ }^{* * * *}$
}

Recibido: 26/08/2011

Aceptado: 07/05/2013

\section{RESUMEN}

Las propiedades físicas y reológicas a altas temperaturas de servicio de un cemento asfáltico (CA) modificado con un desecho de policloruro de vinilo (PVC) fueron evaluadas y son presentadas en el artículo. Adicionalmente se presenta la influencia del tiempo de mezcla del CA con el PVC y el envejecimiento a corto plazo. Un incremento notable en la rigidez y la resistencia a fluir se reporta cuando se adiciona el desecho de PVC a un CA 80-100. De la misma forma, se reporta un incremento en la temperatura máxima de operación en servicio del ligante modificado.

Palabras clave: desecho de PVC, policloruro de vinilo, asfalto modificado, propiedades físicas y reológicas.

Ingeniero civil, M. Sc., Ph. D. Director magíster en Ingeniería Civil, grupo CECATA, Facultad de Ingeniería, Pontificia Universidad Javeriana, Bogotá D. C., Colombia. E-mail: fredy.reyes@javeriana.edu.co

** Ingeniera civil, Investigador Grupo de Investigación CECATA. Departamento de Ingeniería Civil, Facultad de Ingeniería, Pontificia Universidad Javeriana, Bogotá D. C., Colombia. E-mail: cguaqueta@javeriana.edu.co

*** Ingeniera civil, Investigador Grupo de Investigación CECATA. Departamento de Ingeniería Civil, Facultad de Ingeniería, Pontificia Universidad Javeriana, Bogotá D. C., Colombia. E-mail: porrasl@javeriana.edu.co

**** Ingeniero Civil, M. Sc., Ph. D. Profesor asociado, Programa de Ingeniería Civil, Facultad de Ingeniería, Universidad de La Salle, Bogotá D.C., Colombia. E-mail: harondon@unisalle.edu.co 


\section{BEHAVIOR OF AN ASPHALT CEMENT MODIFIED WITH A RESIDUE OF PVC}

\section{ABSTRACT}

Physical and rheological properties under high temperatures of service of modified asphalt cement with a residue of PVC were evaluated. Additionally, the influence of mix temperature and short term aging phenomena is presented. Stiffness and strength to flow increased when is adding PVC to asphalt cement. Maximum performance temperature of service increased when is used the modified asphalt cement.

Key words: PVC residues, polyvinyl chloride, modified asphalt cement, physical and rheological properties. 


\section{INTRODUCCIÓN}

La tecnología de los asfaltos y las mezclas asfálticas modificadas ha sido una técnica ampliamente estudiada y utilizada en el mundo. Con la adición de polímeros al asfalto se modifican las propiedades mecánicas, químicas y reológicas de las mezclas asfálticas. Cuando se utiliza esta tecnología se pretende mejorar el comportamiento que experimentan las mezclas tradicionales cuando son sometidas a diferentes condiciones de carga y del medio ambiente. Por lo general las propiedades que se intentan mejorar son la rigidez, la resistencia bajo carga monotónica, al ahuellamiento, la fatiga, y el envejecimiento, y disminuir la susceptibilidad térmica. Esta tecnología es también frecuente cuando se requiere que la superficie de la carretera posea una vida útil más larga de lo normal, o aplicaciones especializadas en las cuales asfaltos modificados permiten espesores más delgados de capas asfálticas de lo que normalmente se requiere [1].

El documento presenta los resultados experimentales de ejecutar ensayos sobre un cemento asfáltico CA 80-100 modificado con un desecho de policloruro de vinilo (PVC). CA 80-100 se refiere a un cemento asfáltico cuya medida en el ensayo de penetración ASTM D-5 está en un rango entre 80 y 100 décimas de mm. Los ensayos fueron ejecutados con el fin de evaluar las propiedades físicas y reológicas que experimenta el CA 80-100 cuando se le adiciona por vía húmeda el desecho de PVC.

La mayor parte de las investigaciones realizadas en el área de los asfaltos modificados utilizan como agentes modificadores polímeros del tipo elastómero [p.e., 2-8]. Estados del conocimiento sobre el tema pueden ser consultados en Papagiannakis y Lougheed [9], Copeland [10], Yildirim [11] y Rondón et al. [12]. Este tipo de aditivos al ser agregados al asfalto mejoran principalmente el comportamiento resiliente (recuperación elástica) de las mezclas cuando son solicitadas a ciclos de carga y descarga especialmente a altas temperaturas de servicio. Como ya se mencionó, en este estudio se propone modificar el cemento asfáltico con un desecho polimérico del tipo plastomérico como es el PVC. Este aditivo se escogió debido principalmente a

- Este tipo de polímero (plastomero) generalmente produce un incremento en la resistencia mecánica de las mezclas a altas temperaturas, debido a que el asfalto se rigidiza.

- Es un material de alta resistencia térmica y química cuya degradación natural dura más de 100 años [13].

- De acuerdo con Reyes y Figueroa [14], en Bogotá D. C. diariamente se producen alrededor de 600 toneladas de basuras de las cuales el $10 \%$ aproximadamente son plásticos y el consumo per cápita de estos materiales en Colombia es de $11.3 \mathrm{~kg}$ anuales. De este $10 \%$ gran parte proviene de desechos de PVC los cuales pueden ser utilizados para modificar las propiedades de mezclas asfálticas y así disminuir el impacto ambiental negativo que generan.

Para la evaluación de las propiedades físicas del CA convencional y modificado fueron ejecutados ensayos de penetración, punto de ablandamiento y viscosidad. Las propiedades reológicas fueron evaluadas realizando ensayos en un reómetro dinámico de corte (DSR por sus siglas en inglés).

\section{METODOLOGÍA}

Al cemento asfáltico se le realizaron los ensayos típicos que exige la especificación del INVIAS [15] para caracterizarlos como son (ver tabla 1): penetración, viscosidad absoluta, ductilidad, solubilidad en tricloroetileno, contenido de agua, punto de ablandamiento (anillo y bola) y ensayos al residuo, luego del ensayo de película delgada en horno rotatorio (RTFOT por sus siglas en inglés). Estos ensayos fueron ejecutados siguiendo los lineamientos establecidos por el INVIAS [16]. El policloruro de vinilo (PVC) utilizado es un desecho obtenido de la producción de resinas prime 
Tabla 1. Características generales del cemento asfáltico CA 80-100

\begin{tabular}{|l|c|c|c|c|}
\hline \multicolumn{1}{|c|}{ Ensayo } & Método & Unidad & CA 80-100 & Resultado \\
\hline \multicolumn{4}{|c|}{ Ensayos sobre el asfalto original } \\
\hline Penetración $\left(25^{\circ} \mathrm{C}, 100 \mathrm{~g}, 5 \mathrm{~s}\right)$ & ASTM D-5 & $0.1 \mathrm{~mm}$ & $80-100$ & 96 \\
\hline Índice de penetración & INV. E-724 & - & $-1 /+1$ & -0.2 \\
\hline Punto de ablandamiento & INV. E -712 & ${ }^{\circ} \mathrm{C}$ & $42-52$ & 50.6 \\
\hline Viscosidad absoluta $\left(60^{\circ} \mathrm{C}\right)$ & ASTM D-4402 & Poises & 1000 mín. & 1400 \\
\hline Ductilidad $\left(25^{\circ} \mathrm{C} .5 \mathrm{~cm} / \mathrm{min}\right)$ & ASTM D-113 & $\mathrm{Cm}$ & 100 mín. & $>100$ \\
\hline Solubilidad en Tricloroetileno & ASTM D-2042 & $\%$ & 99 mín. & $>99$ \\
\hline Contenido de agua & ASTM D-95 & $\%$ & 0.2 máx. & $<0.2$ \\
\hline Punto de inflamación COC & ASTM D-92 & ${ }^{\circ} \mathrm{C}$ & 232 mín. & 295 \\
\hline \multicolumn{7}{|c|}{ Ensayos sobre el residuo luego del RTFOT } \\
\hline Pérdida de masa & ASTM D-2872 & $\%$ & 1.0 máx. & 0.3 \\
\hline Penetración $\left(25^{\circ} \mathrm{C}, 100 \mathrm{~g}, 5 \mathrm{~s}\right)$ & ASTM D-5 & $\%$ & 48 mín. & 65 \\
\hline
\end{tabular}

Fuente: elaboración propia

de PVC; presenta una densidad de $0.9 \mathrm{~g} / \mathrm{cm}^{3} \mathrm{y}$ partículas de coloración blanca que pasan el tamiz N. 200 en un ensayo de granulometría cuando el material está en estado seco.

Luego de realizar los ensayos al cemento asfáltico (CA), se mezcló por vía húmeda el $\mathrm{CA}$ y el desecho de $\mathrm{PVC}$ en relaciones de $\mathrm{PVC} / \mathrm{CA}=5$, $10,15,20$ y $25 \%$ a una temperatura de $155^{\circ} \mathrm{C}$. Esta temperatura fue escogida debido a que por encima de la misma el CA experimenta envejecimiento por pérdida de componentes químicos por oxidación, y por debajo, el mezclado se dificulta especialmente cuando el contenido de PVC es alto. Los tiempos de mezclado fueron de 20 y 40 minutos. Esta temperatura y tiempos de mezcla son similares a aquellos reportados por otros investigadores cuando han modificado CA con aditivos plastoméricos (por ejemplo, [17]). Para la mezcla de los componentes se utilizó el equipo mecánico ULTRA TURRAX T50.

Para evaluar las propiedades físicas del cemento asfáltico modificado se realizaron ensayos de penetración (a la temperatura estándar de $25^{\circ} \mathrm{C}$ ) y punto de ablandamiento. Adicionalmente, se realizaron ensayos de viscosidad con el fin de determinar las temperaturas de mezcla y compactación. Las propiedades reológicas fueron evaluadas por medio de un DSR. El módulo complejo de corte $\left(G^{*}\right)$ y el ángulo de fase $(\delta)$ fueron obtenidos bajo temperaturas intermedias y altas de servicio del CA: $40,44,48,52,56,60$ y $64^{\circ} \mathrm{C}$. La frecuencia de aplicación del esfuerzo de corte fue de $1.59 \mathrm{~Hz}$. Los ensayos fueron ejecutados con base en la forma como lo recomienda el Instituto del Asfalto [18]. Para evaluar el efecto del envejecimiento a corto plazo, de acuerdo con la especificación ASTM D-2872, sobre las propiedades reológicas de los CA, fueron ejecutados ensayos en un horno rotatorio de película delgada (RTFOT por sus siglas en inglés).

Para el análisis de los resultados obtenidos de los ensayos no se elaboraron muestras con PVC/ $\mathrm{CA}=25 \%$ debido a que cuando se realizó el proceso de mezclado se notó que la cantidad de PVC era tan elevada que impedía que se desarrollara una mezcla relativamente homogénea y el aditivo generaba grumos en la muestra. Adicionalmente, las temperatura de mezcla y compactación fueron muy elevadas lo que genera un rápido envejeci- 
miento del ligante asfáltico durante su fabricación, y dificultad en los procesos de construcción de la mezcla en obra.

\section{RESULTADOSY DISCUSIÓN}

\subsection{Propiedades físicas}

En las figuras 1 y 2 se presentan los resultados de los ensayos de penetración y punto de ablandamiento realizados sobre el CA convencional y modificado. Adicionalmente se presenta la influencia del tiempo de mezclado. En las figuras se observa que el asfalto modificado presenta una resistencia a la penetración y a fluir, superior con respecto al convencional (CA 80-100 sin modificar) para cualquier porcentaje PVC/CA. La mayor resistencia a la penetración y temperatura a la cual comienza a fluir el material se obtiene cuando se utiliza una relación PVC/CA de $15 \%$. Con esta relación se observa una disminución de $59.2 \%$ y $74.9 \%$ en los valores de penetración con respecto al CA convencional cuando estos materiales son mezclados durante 20 y 40 minutos, respectivamente. De la misma forma, el incremento del punto de ablandamiento en este contenido de PVC y CA es de $36.6 \%$ y $40.4 \%$. Se observa adicionalmente un incremento en la penetración y disminución en el punto de ablandamiento cuando se aumenta el porcentaje de adición de PVC desde PVC/CA=15 $\%$ hasta PVC/CA=20\%. Este comportamiento se debe a que la cantidad de PVC en PVC/CA=20\% es tan elevada que el aditivo no se alcanza a disolver lo suficiente en el CA. Incrementando el tiempo de mezclado del CA con el PVC se obtiene un material con mayor resistencia a la penetración y a fluir, debido principalmente a que el aumento en el tiempo de mezcla genera una mayor disolución del aditivo en el CA.

Cuando se modifica el CA con el PVC, el material experimenta un aumento en su pérdida de masa en el ensayo de envejecimiento a corto plazo utilizando el RTFOT; este aumento es menor a medida que incrementa el tiempo de mezclado y disminuye la cantidad de PVC adicionado. Lo anterior significa que esta pérdida de masa se obtiene principalmente en el desecho de PVC y es mayor cuando no se obtiene un mezclado homogéneo entre ambos materiales. La pérdida de masa máxima permitida por especificación (1\%) se obtiene

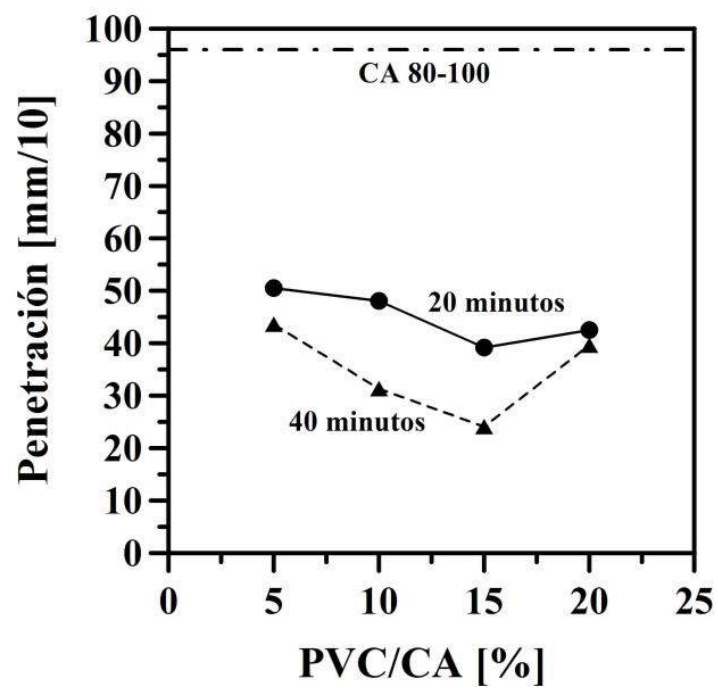

Figura 1. Evolución de la penetración con la relación PVC/CA y el tiempo de mezclado

Fuente: elaboración propia

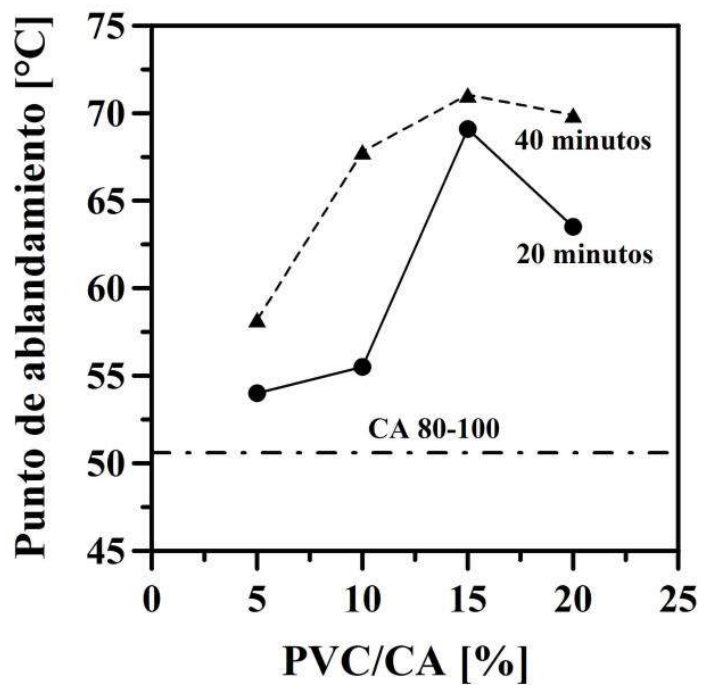

Figura 2. Evolución del punto de ablandamiento con la relación PVC/CA y el tiempo de mezclado

Fuente: elaboración propia 
cuando se mezclan 40 minutos los materiales y se adicionan en proporciones PVC/CA=5, $10 \%$.

El aumento en la viscosidad del CA al ser modificado con PVC genera incremento en las temperaturas de fabricación de las mezclas, y de extensión y compactación (ver tabla 2).

\subsection{Propiedades reológicas}

En las tablas 3 y 4 se presentan los resultados de evolución del $G^{*}$ y $\delta$ con la temperatura $(T)$ de ensayo en el DSR sobre las muestras sin y con envejecimiento en RTFOT, respectivamente. Se observa un aumento y disminución típico del $\mathrm{G}^{*}$ y $\delta$, respectivamente, a medida que disminuye la temperatura de ensayo y se envejecen las muestras. Con el fin de evaluar las propiedades reológicas a altas temperaturas de servicio, en las figuras 4 y 5 se presenta la evolución de $G^{*}$ y $\delta$ con la temperatura máxima del ensayo $\left(64^{\circ} \mathrm{C}\right)$. Lo ideal a altas temperaturas para evitar el fenómeno de ahuellamiento de mezclas asfálticas es que la relación $G^{*} / \operatorname{sen}(\delta)$ sea lo más alta posible porque lo que se requiere

Tabla 2. Temperaturas de mezcla y compactación para CA 80-100 convencional y modificado con PVC

\begin{tabular}{|c|c|c|}
\hline $\begin{array}{c}\text { PVC/CA } \\
{[\%]}\end{array}$ & $\begin{array}{c}\text { Temperatura } \\
\text { de mezcla }\left[{ }^{\circ} \mathrm{C}\right]\end{array}$ & $\begin{array}{c}\text { Temperatura de } \\
\text { compactación }\left[{ }^{\circ} \mathrm{C}\right]\end{array}$ \\
\hline \multicolumn{3}{|c|}{20 minutos de mezcla } \\
\hline 0 & 138 & 136 \\
\hline 5 & 140 & 138.5 \\
\hline 10 & $>165$ & 151 \\
\hline 15 & $>165$ & 164 \\
\hline 20 & $>165$ & 136 \\
\hline & 40 minutos de mezcla \\
\hline 0 & 138 & 137.9 \\
\hline 5 & $>165$ & 148.5 \\
\hline 10 & $>165$ & 161 \\
\hline 15 & $>165$ & 159.5 \\
\hline 20 & & \\
\hline
\end{tabular}

Fuente: elaboración propia es un material rígido y elástico (mayor $\mathrm{G}^{*}$ y menor $\delta)$. Los resultados son consistentes con aquellos obtenidos en los ensayos de penetración y punto de ablandamiento. A medida que incrementa la relación PVC/CA aumenta la magnitud de $G^{*}$

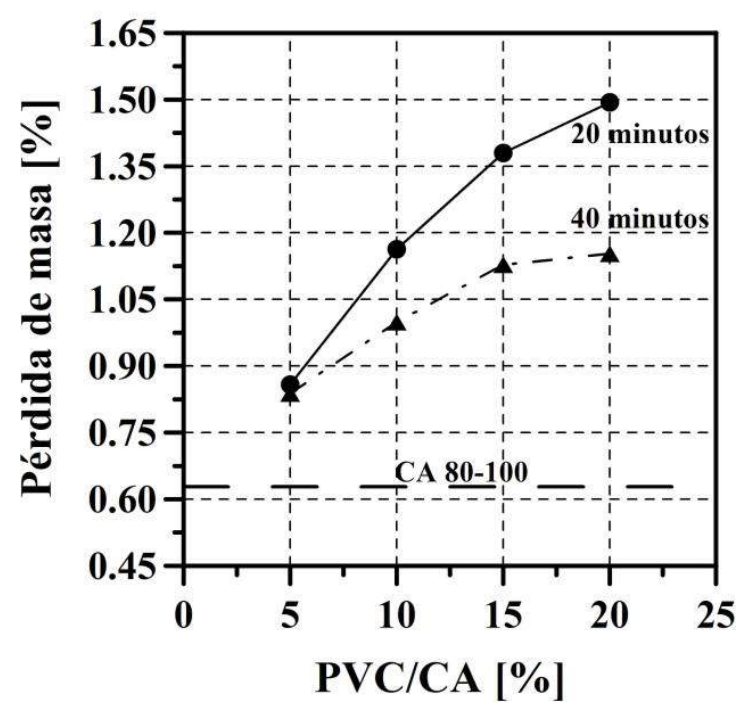

Figura 3. Evolución de la pérdida de masa con la relación PVC/CA y el tiempo de mezclado

Fuente: elaboración propia

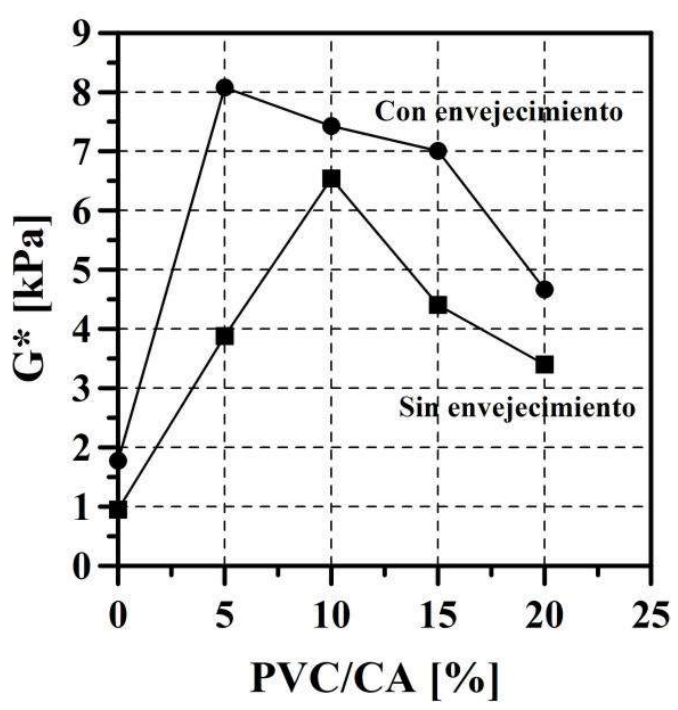

Figura 4. Evolución de $\mathrm{G}^{*}$ con la relación PVC/CA para muestras sin y con envejecimiento en RTFOT y temperatura de $64^{\circ} \mathrm{C}$

Fuente: elaboración propia 
Tabla 3. Evolución de los parámetros reológicos de las muestras sin envejecimiento en RTFOT

\begin{tabular}{|c|c|c|c|c|c|c|c|c|c|c|}
\hline \multicolumn{11}{|c|}{$\mathrm{PVC} / \mathrm{CA}[\%]$} \\
\hline \multirow[b]{2}{*}{$\mathrm{T}\left[{ }^{\circ} \mathrm{C}\right]$} & \multicolumn{2}{|c|}{0} & \multicolumn{2}{|c|}{5} & \multicolumn{2}{|c|}{10} & \multicolumn{2}{|c|}{15} & \multicolumn{2}{|c|}{20} \\
\hline & $\begin{array}{c}\mathrm{G}^{*} \\
{[\mathrm{kPa}]}\end{array}$ & $\begin{array}{c}\delta \\
{\left[{ }^{\circ}\right]}\end{array}$ & $\begin{array}{c}\mathrm{G}^{*} \\
{[k \mathrm{kPa}]}\end{array}$ & $\begin{array}{c}\delta \\
{\left[{ }^{\circ}\right]}\end{array}$ & $\begin{array}{c}\mathrm{G}^{*} \\
{[k \mathrm{~Pa}]}\end{array}$ & $\begin{array}{c}\delta \\
{\left[{ }^{\circ}\right]}\end{array}$ & $\begin{array}{c}\mathrm{G}^{*} \\
{[\mathrm{kPa}]}\end{array}$ & $\begin{array}{c}\delta \\
{\left[{ }^{\circ}\right]}\end{array}$ & $\begin{array}{c}\mathrm{G}^{*} \\
{[k \mathrm{kPa}]}\end{array}$ & $\begin{array}{c}\delta \\
{\left[{ }^{\circ}\right]}\end{array}$ \\
\hline 40 & 29.96 & 81.9 & 93.04 & 69.6 & 115.34 & 65.3 & 93.06 & 69.3 & 76.94 & 71.0 \\
\hline 44 & 15.52 & 83.5 & 56.04 & 71.3 & 77.69 & 66.7 & 56.60 & 70.8 & 45.40 & 72.8 \\
\hline 48 & 8.39 & 84.7 & 32.63 & 73.3 & 48.71 & 68.5 & 33.90 & 72.6 & 26.69 & 74.8 \\
\hline 52 & 4.68 & 85.8 & 18.93 & 75.4 & 29.79 & 70.6 & 20.22 & 74.6 & 15.69 & 76.9 \\
\hline 56 & 2.67 & 86.7 & 11.04 & 77.5 & 17.99 & 73.0 & 12.05 & 76.7 & 9.30 & 78.9 \\
\hline 60 & 1.57 & 87.4 & 6.50 & 79.5 & 10.80 & 75.4 & 7.22 & 78.8 & 5.56 & 80.9 \\
\hline 64 & 0.95 & 88 & 3.88 & 81.2 & 6.54 & 77.5 & 4.41 & 80.5 & 3.40 & 82.5 \\
\hline
\end{tabular}

Fuente: elaboración propia

Tabla 4. Evolución de los parámetros reológicos de las muestras con envejecimiento en RTFOT

\begin{tabular}{|c|c|c|c|c|c|c|c|c|c|c|}
\hline \multicolumn{11}{|c|}{ PVC/CA [\%] } \\
\hline \multirow[b]{2}{*}{$\mathrm{T}\left[{ }^{\circ} \mathrm{C}\right]$} & \multicolumn{2}{|c|}{0} & \multicolumn{2}{|c|}{5} & \multicolumn{2}{|c|}{10} & \multicolumn{2}{|c|}{15} & \multicolumn{2}{|c|}{20} \\
\hline & $\begin{array}{c}\mathrm{G}^{*} \\
{[\mathrm{kPa}]}\end{array}$ & $\begin{array}{c}\delta \\
{\left[{ }^{\circ}\right]}\end{array}$ & $\begin{array}{c}\mathrm{G}^{*} \\
{[\mathrm{kPa}]}\end{array}$ & $\begin{array}{c}\delta \\
{\left[{ }^{\circ}\right]}\end{array}$ & $\begin{array}{c}\mathrm{G}^{*} \\
{[\mathrm{kPa}]}\end{array}$ & $\begin{array}{c}\delta \\
{\left[{ }^{\circ}\right]}\end{array}$ & $\begin{array}{c}\mathrm{G}^{*} \\
{[\mathrm{kPa}]}\end{array}$ & $\begin{array}{c}\delta \\
{\left[{ }^{\circ}\right]}\end{array}$ & $\begin{array}{c}\mathrm{G}^{*} \\
{[\mathrm{kPa}]}\end{array}$ & $\begin{array}{c}\delta \\
{\left[{ }^{\circ}\right]}\end{array}$ \\
\hline 40 & 60.11 & 77.4 & 149.88 & 65.1 & 132.14 & 66.5 & 95.39 & 69.0 & 100.51 & 71.3 \\
\hline 44 & 31.64 & 79.4 & 98.17 & 66.3 & 87.47 & 67.7 & 59.02 & 70.6 & 59.64 & 72.8 \\
\hline 48 & 16.91 & 81.3 & 60.84 & 67.9 & 54.39 & 69.3 & 36.38 & 72.4 & 35.23 & 74.4 \\
\hline 52 & 9.30 & 83.0 & 36.84 & 69.8 & 33.06 & 71.1 & 22.75 & 74.4 & 20.90 & 76.2 \\
\hline 56 & 5.23 & 84.4 & 22.15 & 72.0 & 19.99 & 73.1 & 14.61 & 76.5 & 12.50 & 78.1 \\
\hline 60 & 3.01 & 85.5 & 13.27 & 74.3 & 12.09 & 75.2 & 9.80 & 78.6 & 7.55 & 79.9 \\
\hline 64 & 1.78 & 86.5 & 8.08 & 76.4 & 7.43 & 77.2 & 7.01 & 80.3 & 4.67 & 81.3 \\
\hline
\end{tabular}

Fuente: elaboración propia

hasta un valor máximo a partir del cual disminuye, principalmente porque el desecho de PVC no se alcanza a disolver lo suficiente en el CA. Los mayores y menores valores de $G^{*}$ y $\delta$, respectivamente, se obtienen cuando se utilizan relaciones de PVC/ CA entre 5 a $10 \%$ para cualquier temperatura de ensayo entre 40 a $64{ }^{\circ} \mathrm{C}$ (ver figuras 6 y 7 ).

La evolución de los parámetros reológicos con la temperatura puede ser descrita matemáticamente a través de la ecuación (1). El coeficiente de correlación cuando se utiliza la ecuación (1) es $\mathrm{r}^{2}=0.99 \mathrm{y}$ $a, b$ son variables de estado que varían entre -2.63 a -4.0 y $1.86 \times 10^{4}$ a $2.78 \times 10^{4}$, respectivamente, dependiendo de la temperatura de ensayo. Utilizando esta ecuación es posible determinar las temperaturas máximas de funcionamiento del ligante asfáltico convencional y modificado. Se observa en la figura 8, que el asfalto modificado puede ser utilizado a 
mayor temperatura con respecto al convencional para cualquier porcentaje de PVC adicionado. Las mayores temperaturas de funcionamiento $\left(T_{\max }\right)$ del ligante modificado se obtienen cuando la relación PVC/CA es del $10 \%\left(72^{\circ} \mathrm{C}\right)$. En este porcentaje,

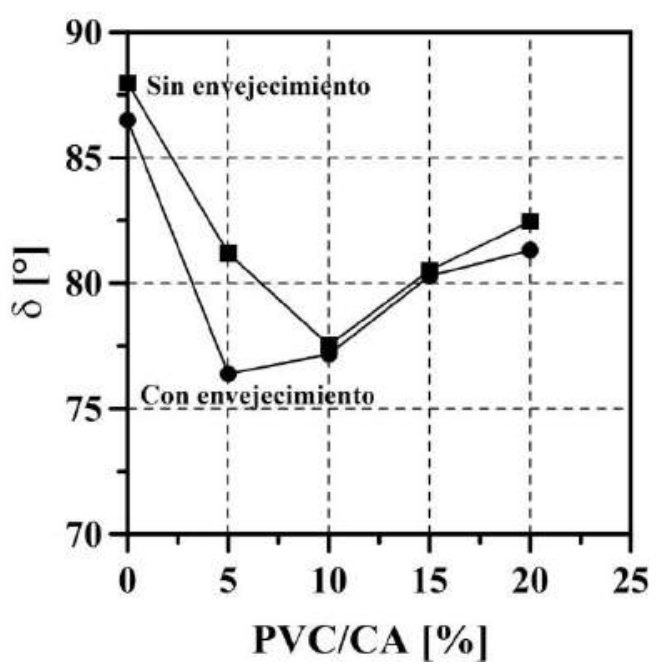

Figura 5. Evolución de $\delta$ con la relación PVC/CA para muestras sin y con envejecimiento en RTFOT y temperatura de $64^{\circ} \mathrm{C}$

Fuente: elaboración propia

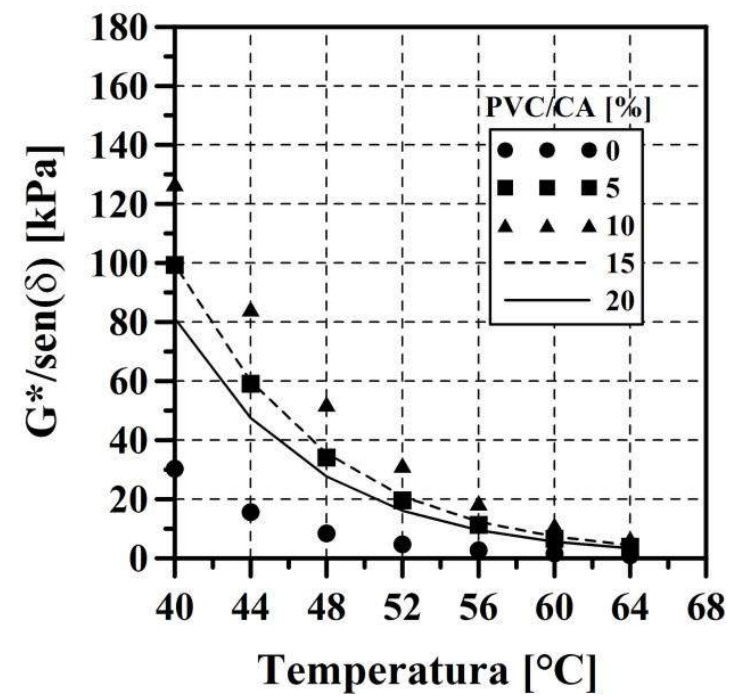

Figura 6. Evolución de $\mathrm{G}^{*} / \operatorname{sen}(\delta)$ con el porcentaje de adición de PVC/CA y la temperatura sobre muestras sin envejecer

Fuente: elaboración propia el incremento en $T_{\max }$ con respecto al convencional es del $16 \%$.

$$
\frac{G^{*}}{\operatorname{sen}(\delta)}=\left(a+\frac{b}{T^{2}}\right)^{2}
$$

\section{CONCLUSIONES}

En el presente estudio se evaluó la influencia que tiene, sobre las propiedades físicas y reológicas a altas temperaturas de servicio, adicionar un desecho de PVC a un CA 80-100. Cuando se modifica el CA con el PVC la resistencia a la penetración, viscosidad y el punto de ablandamiento aumentan debido principalmente a que el aditivo rigidiza el ligante. Estos cambios en las propiedades físicas fueron consistentes con aquellos obtenidos cuando se evaluaron las propiedades reológicas a altas temperaturas de servicio. El mejor comportamiento del asfalto modificado se obtuvo cuando se adicionó el PVC en una proporción de $\mathrm{PVC} / \mathrm{CA}=$ $10 \%$ y se mezcló durante 40 minutos con el CA. En este porcentaje la temperatura máxima de funcionamiento del ligante para evitar el fenómeno de ahuellamiento incrementa en un $16 \%$.

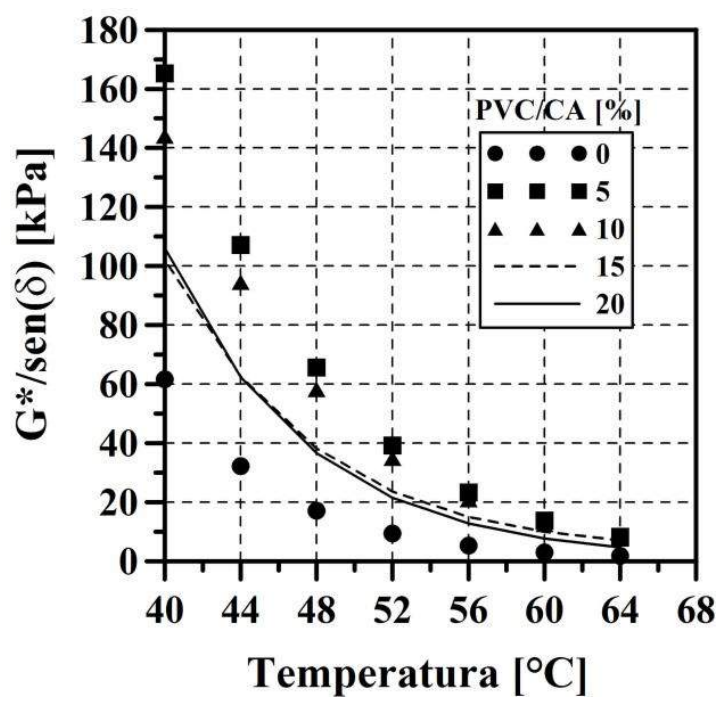

Figura 7. Evolución de $G^{*} / \operatorname{sen}(\delta)$ con el porcentaje de adición de PVC/CA y la temperatura sobre muestras con envejecimiento

Fuente: elaboración propia 
Con los resultados obtenidos, se recomienda la modificación de CA con desecho de PVC en zonas con condiciones de temperaturas medias anuales promedios (TMAP) altas. El incremento en viscosidad y rigidez del ligante, y la evolución de los parámetros reológicos con el porcentaje de aditivo y la temperatura permiten prever su utilización en vías para altos volúmenes de tránsito y/o zonas con TMAP elevadas donde se presenten problemas de ahuellamiento.

En general, el CA modificado con desecho de PVC, tiende a poseer un comportamiento rígido. A bajas temperaturas de servicio estas mezclas pueden tener un comportamiento frágil, llevando a pensar que tendrían un mejor desempeño en climas cálidos. Las fases futuras del proyecto deben medir propiedades como reología a bajas temperaturas de servicio y envejecimiento a largo plazo de los asfaltos para evaluar la resistencia al fenómeno de fatiga. Adicionalmente, para entender los cambios que ocurren en las propiedades físicas y reológicas del asfalto modificado es necesario evaluar los cambios que experimentan en sus propiedades químicas.

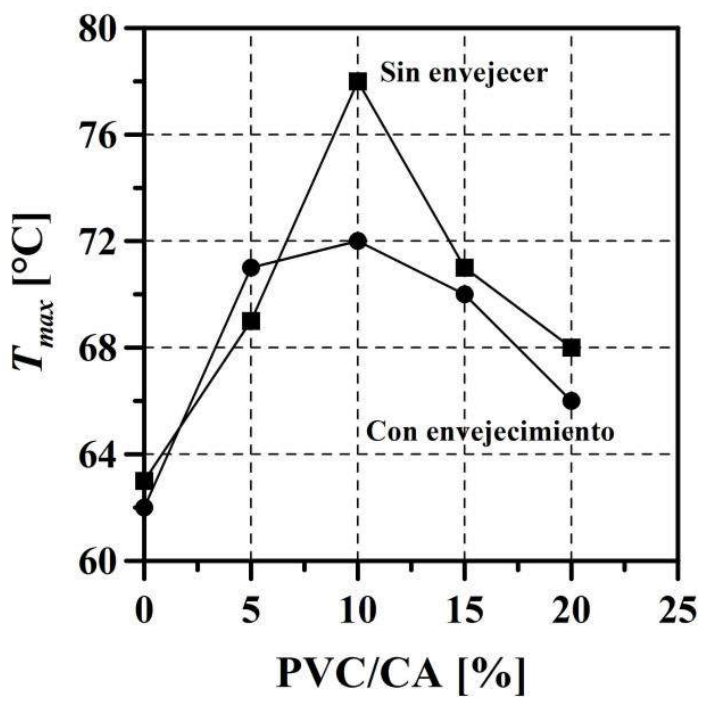

Figura 8. Evolución de la temperatura máxima de funcionamiento Tmax con la relación PVC/CA sobre las muestras con y sin envejecimiento

Fuente: elaboración propia

\section{REFERENCIAS}

[1] D. Casey, C. McNally, A. Gibneya and M. Gilchrist, "Development of a recycled polymer modified binder for use in stone mastic asphalt", Journal of Resources, Conservation and Recycling, Vol. 52, pp. 1167-1174, 2008.

[2] J-S. Chen, M-C. Liao and M-S. Shiah, "Asphalt modified by Styrene-Butadiene-Styrene triblock copolymer: morphology and model”. J. Material in Civil Eng., Vol. 14, N. 3, pp. 224-229, 2002.

[3] J. L. McQuillen, H. B. Takallou, R. G. Hicks, and D. Esch, "Economic analysis of rubber-modified asphalt mixes", J. Transportation Engineering, Vol. 114, N. ${ }^{\circ}$, pp. 259-277, 1988.

[4] S-J. Lee, S-N. Amirkhanian, K. Shatanawi and C. Thodesen, "Influence of compaction temperature on rubberized asphalt mixes and binders", Canadian J. Civil Eng., Vol. 35, N. 9, pp. 908-917, 2008.

[5] F. Olivares, B. Schultz, M. Fernández and B. Moro, "Rubber-modified hot-mix asphalt pavement by dry process”, Int. J. Pavement Engineering, Vol. 10, N. ${ }^{\circ}$, pp. 277-288, 2009.

[6] J. Stastna, L. Zanzotto and O. Vacin, "Damping of shear vibrations in asphalt modified with styrenebutadiens-styrene polymer", Transportation Research Record, Journal of TRB, N. 1728 , pp. 15-20, 2000.

[7] J. Stastna, L. Zanzotto and O. Vacin, "Viscosity function in polymer-modified asphalts", Journal of Colloid and Interface Science, Vol. 259, N.` 1, pp. 200-207, 2003.

[8] Instituto de Desarrollo Urbano - IDU, "Especificación técnica para la aplicación del grano de caucho reciclado (GCR) en mezclas asfálticas en caliente (vía húmeda)”, Bogotá D.C., Colombia, 2009.

[9] A. T. Papagiannakis and T. J. Lougheed, "A review of crumb-rubber modified asphalt concrete technology". Research Report for Project T9902-09 "Rubber-Asphalt Study”, Washington State Transportation Commission and U. S. Department of Transportation, 1995.

[10] A. R. Copeland, Jr, J. S. Youtcheff and A. Shenoy, "Moisture sensitivity of modified asphalt binders: factors influencing bond strength", Transportation Research Record: Journal of TRB, N. 1998 , pp. 1828, 2007.

[11] Y. Yildirim, "Polymer modified asphalt binders", Construction and Building Materials, Vol. 21, N. ${ }^{\circ}$, pp. 66-72, 2007.

[12] H. A. Rondón, F. A. Reyes, A. S. Figueroa, E. Rodríguez, C. M. Real y T. A. Montealegre, "Mezclas asfálticas 
modificadas en Colombia”, Revista Infraestructura Vial, N. ${ }^{\circ}$ 19, pp. 12-21, 2008.

[13] CAG - Ciudadanía Ambiental Global, "Basura al aire libre”. http://www.cag.org.mx/16.html. Acceso 12 Octubre 2009.

[14] F. A. Reyes y A. F. Figueroa, "Uso de desechos plásticos en mezclas asfálticas - Síntesis de la investigación colombiana", Ed. Pontificia U. Javeriana, Bogotá D.C. (Colombia), 103 p., 2008.

[15] Instituto Nacional de Vías - INVIAS, "Especificaciones generales de construcción de carreteras", Bogotá D. C., Colombia, 2007.
[16] Instituto Nacional de Vías - INVIAS, "Normas de ensayos de materiales para carreteras", V. I y II, Bogotá D.C., Colombia, 2007.

[17] C. Fang, T. Li, Z. Zhang and D. Ping, "Modification of asphalt by packaging waste-polyethylene", Polymer Composites, Vol. 29, ․․ 5, pp. 500-505, 2008.

[18] Instituto del Asfalto, "Performance Graded Asphalt Binder Specification and Testing", SP-1, pp. 6-25, 1998. 